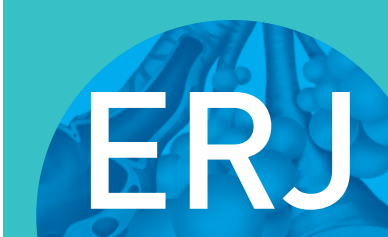

open research

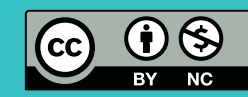

\title{
The benefits of pulmonary rehabilitation in patients with COVID-19
}

\author{
Zhen-feng $\mathrm{He}^{1}$, Nan-shan Zhong ${ }^{1,2}$ and Wei-jie Guan, ${ }^{1,2}$ \\ ${ }^{1}$ State Key Laboratory of Respiratory Disease, National Clinical Research Center for Respiratory Disease, \\ Guangzhou Institute of Respiratory Health, First Affiliated Hospital of Guangzhou Medical University, \\ Guangzhou Medical University, Guangzhou, China. ${ }^{2}$ Both authors contributed equally to the work.
}

Correspondence: Wei-jie Guan, State Key Laboratory of Respiratory Disease, National Clinical Research Center for Respiratory Disease, Guangzhou Institute of Respiratory Disease, First Affiliated Hospital of Guangzhou Medical University, 151 Yanjiang Road, Guangzhou, Guangdong, China.

E-mail: battery203a163.com

@ERSpublications

This editorial reviews the evidence supporting benefits of pulmonary rehabilitation in \#COVID19 patients, as well as some unanswered research questions https://bit.ly/39JY3SU

Cite this article as: He Z-f, Zhong N-s, Guan W-j. The benefits of pulmonary rehabilitation in patients with COVID-19. ERJ Open Res 2021; 7: 00212-2021 [https://doi.org/10.1183/ 23120541.00212-2021].

As of 10 March 2021, coronavirus disease 2019 (COVID-19) has resulted in more than 0.1 billion laboratory-confirmed cases and more than 2.5 million deaths globally [1]. Both the economic impact and disease burden of COVID-19 far exceeded those of severe acute respiratory syndrome and Middle East Respiratory Syndrome. The number of patients who have recovered from COVID-19 is increasing [2] despite an escalation of total number of cases worldwide.

The clinical manifestations of COVID-19 were heterogeneous, ranging from asymptomatic or mild diseases that did not require any specific medical treatment (accounting for $\sim 80 \%$ of patients) to the critical illness which rapidly progressed to death (accounting for $\sim 3 \%$ of patients) [3]. In many patients, COVID-19 was typically characterised by cough, dyspnoea and fever on hospital admission. Other symptoms such as fatigue, muscle weakness and dysgeusia have also been reported [4]. Unfortunately, a considerable proportion of patients had residual symptoms (including cough, fatigue, muscle weakness and mental symptoms) on discharge from hospital, and some of these symptoms persisted for at least a median of 6 months of follow-up [5]. Furthermore, the severity of COVID-19 correlated with the likelihood of having residual symptoms and abnormal lung function [5-7]. These findings were in line with the results from the long-term follow-up study among patients with severe acute respiratory syndrome which demonstrated that approximately $40 \%$ of these survivors still experienced chronic fatigue for a mean of 41.3 months [8].

Given the growing number of patients recovering from COVID-19 and the notable long-lasting adverse consequences, it is crucial for clinicians to explore novel approaches to help patients ameliorate the residual symptoms. Pulmonary rehabilitation (PR), which includes (but is not limited to) exercise training, education, and behavioural changes, might have a role in accelerating the improvement in the physical and psychological condition of patients with COVID-19 after discharge from hospital [9]. Indeed, several studies have also documented the therapeutic benefits of PR on improving the quality of life (QoL), respiratory function and psychological parameters of patients with COPD, asthma and lung cancer after thoracic surgery [10-12].

In the context of the global management of the aftermath of COVID-19, there remains a lack of evidence to verify the safety and efficacy of PR on patients discharged from hospital, although some expert 
consensus and guidelines have been published by the World Health Organization [13], Chinese Medical Association of Rehabilitation [14], and European Respiratory Society/American Thoracic Society [15]. These documents recommended early bedside in-hospital rehabilitation and regular daily activities after hospital discharge among patients with COVID-19 [15]. However, in light of the paucity of studies, no specific recommendations have been made regarding the optimal mode, intensity, duration and course of PR. It was believed that the implementation of a comprehensive PR programme would be superior to no PR programme in accelerating the recovery of COVID-19 [15].

In this issue of ERJ Open Research, GLOeCKL et al. [16] evaluated the efficacy, feasibility and safety of an integrated PR programme in COVID-19 patients with different grading of disease severity. The study prospectively evaluated the improvement of exercise capacity, lung function, QoL and psychological impairment among 50 patients (24 with mild/moderate COVID-19 and 26 with severe/critical illness of COVID-19) after a 3-week supervised PR programme, which was similar to that intended for patients with lung fibrosis, in a rehabilitation centre. The medium duration between the first positive PCR assay and PR administration was 178 days and 61 days for the mild/moderate group and the severe/critical illness group, respectively. At the end of follow-up, there was a significant improvement in the 6-min walking distance (6 MWD), forced vital capacity, forced expiratory volume in $1 \mathrm{~s}$, and short-form 36 questionnaire (SF-36) total score in both groups. Importantly, the severe/critical illness group benefited significantly from the improvement in the mental health component of SF-36. No adverse event was recorded during the PR. Moreover, a numerically but not significantly lower number of patients reported pre-existing symptoms such as dyspnoea, fatigue or cough after PR. Therefore, PR was deemed effective and safe to improve exercise performance, lung function and QoL in COVID-19 patients with different grading of disease severity.

The study by GloeckL et al. [16] has added to the accumulating evidence regarding the role PR plays in accelerating the recovery of COVID-19. Several studies have reported certain benefits associated with the intervention of a short course of PR. For instance, both lower limb strength and cardiopulmonary endurance could be improved significantly after a supervised 2-week out-patient PR programme via the telehealth platform (65\% of the 44 patients achieved the clinically meaningful difference) or home-based exercise programme ( $88 \%$ of the 25 patients achieved the clinically meaningful difference) [17]. A 28-day course of PR also markedly increased the $6 \mathrm{MWD}$ in 21 patients with COVID-19 who were discharged from the intensive care unit [18]. In a prospective clinical trial that recruited 72 elderly patients with COVID-19 who were randomly assigned to receive either a 6-week PR programme or usual care alone, PR resulted in a marked improvement in the lung function, QoL and anxiety, but not depression [19]. Furthermore, a 20-day in-patient cardiopulmonary rehabilitation programme for 28 patients with COVID-19 in a general ward significantly improved the $6 \mathrm{MWD}$ by a mean of $130 \mathrm{~m}$ as well as the health status [20]. Therefore, most studies found that PR consistently accelerated the recovery of physical function but the impact on mental health remains elusive. Indeed, the adverse impact of an epidemic outbreak such as COVID-19 and severe acute respiratory syndrome on mental health might persist longer than that on the physical health $[5,8]$. Despite the lack of a control group, findings of the study by GLOECKL et al. [16] have also shed light on the positive role of PR in accelerating the recovery of mental health which has been an important neglected issue.

However, there remain some unanswered research questions. In light of the self-limited nature of COVID-19 among most of the patients, the improvement in 6 MWD might have been confounded by the rate of the natural recovery of COVID-19. DAHER et al. [21] recruited a cohort of patients with severe COVID-19 who did not receive a PR programme, and reported a median of $380 \mathrm{~m}$ on the $6 \mathrm{MWD}$ after $\sim 56$ days of discharge from hospital which was significantly lower than the median of $468 \mathrm{~m}$ reported by GLOECKL et al. [16]. It was likely that the improvements in patients recovering from severe COVID-19 might be attributed to the addition of a PR programme. In addition, the PR programme varied considerably from one to other, which precluded any direct comparison of the efficacy across different study designs. However, the heterogeneity of COVID-19 also indicated the need to implement an individualised PR programme [22]. PR should ideally integrate respiratory muscle training, cough exercise, diaphragmatic training, stretching exercise and home exercise, with the assessment of respiratory function, exercise endurance, strength training, QoL and psychological health [19]. The typical duration of the PR programme should persist for at least 6-8 weeks [15]. Furthermore, the implementation of the PR programmes should be adapted to the local situations, taking into account the patient's preference and culture. Table 1 demonstrates the proposed interim instructions from the World Health Organization and European Respiratory Society/American Thoracic Society on PR practice in the hospital and post-hospital phase in COVID-19 patients based on empirical evidence.

Despite the growing understanding on the role of $\mathrm{PR}$, the generalisability of $\mathrm{PR}$ remains to be tested by well-designed randomised controlled trials in larger settings. We keenly await more solid evidence which 
TABLE 1 Interim instructions on pulmonary rehabilitation practice in the acute, sub-acute and long-term phase in coronavirus disease 2019 patients

\begin{tabular}{l|c|c|} 
Phase of care & \multicolumn{1}{c}{ Pulmonary rehabilitation instruction } & Typical delivery setting \\
\hline Acute & $\begin{array}{c}\text { In the acute phase, severe patients receiving } \\
\text { ventilatory support should receive pulmonary } \\
\text { rehabilitation to maintain and improve basic } \\
\text { respiratory function }\end{array}$ & Intensive care units \\
Sub-acute & $\begin{array}{c}\text { In the early recovery period, hospitalised patients } \\
\text { should receive pulmonary rehabilitation which aims } \\
\text { to promote independence with activities of daily } \\
\text { living, and to provide with the psychosocial support } \\
\text { After hospital discharge, patients should receive } \\
\text { pulmonary rehabilitation to recover from physical, } \\
\text { psychological and respiratory impairments in the } \\
\text { first 6-8 weeks }\end{array}$ & $\begin{array}{c}\text { Rehabilitation centres, outpatient } \\
\text { programmes, in-home services, } \\
\text { telehealth }\end{array}$ \\
&
\end{tabular}

will facilitate the implementation of PR in routine clinical practice, which aims to promote the physical and mental health in long-term follow-up for COVID-19 patients.

Acknowledgement: We thank Shan-shan Zha (Shenzhen People's Hospital) and Li-li Guan (Guangzhou Institute for Respiratory Health) for their valuable suggestions.

Author contributions: Zhen-feng He and Wei-jie Guan drafted the manuscript; Wei-jie Guan and Nan-shan Zhong critically revised the manuscript. All authors have approved the final submission.

Conflict of interest: None declared.

Support statement: This work was supported by the Guangzhou Institute for Respiratory Health Open Project (funded by China Evergrande Group) project numbers 2020GIRHHMS09 and 2020GIRHHMS19 (to W-j. Guan), the Zhongnanshan Medical Foundation of Guangdong Province (to W-j. Guan) and the Penghua Care Fund to the Medical Pioneers against Covid-19 of Shenzhen Social Commonwealth Foundation (to W-j. Guan). Funding information for this article has been deposited with the Crossref Funder Registry.

\section{References}

1 World Health Organization. Coronavirus disease (COVID-19) pandemic. www.who.int/emergencies/diseases/ novel-coronavirus-2019 Date last accessed: 10 March 2021.

2 Gautam AP, Arena R, Dixit S, et al. Pulmonary rehabilitation in COVID-19 pandemic era: the need for a revised approach. Respirology 2020; 25: 1320-1322.

3 Guan WJ, Ni ZY, Hu Y, et al. Clinical characteristics of coronavirus disease 2019 in China. N Engl J Med 2020; 382: $1708-1720$.

4 Giacomelli A, Pezzati L, Conti F, et al. Self-reported olfactory and taste disorders in patients with severe acute respiratory coronavirus 2 infection: a cross-sectional study. Clin Infect Dis 2020; 71: 889-890.

5 Huang C, Huang L, Wang Y, et al. 6-month consequences of COVID-19 in patients discharged from hospital: a cohort study. Lancet 2021; 397: 220-232.

6 Xiong $\mathrm{Q}, \mathrm{Xu} \mathrm{M}$, Li J, et al. Clinical sequelae of COVID-19 survivors in Wuhan, China: a single-centre longitudinal study. Clin Microbiol Infect 2021; 27: 89-95.

7 Carfi A, Bernabei R, Landi F. Persistent symptoms in patients after acute COVID-19. JAMA 2020; 324: 603-605.

8 Lam MH, Wing YK, Yu MW, et al. Mental morbidities and chronic fatigue in severe acute respiratory syndrome survivors: long-term follow-up. Arch Intern Med 2009; 169: 2142-2147.

9 Spruit MA, Singh SJ, Garvey C, et al. An official American Thoracic Society/European Respiratory Society statement: key concepts and advances in pulmonary rehabilitation. Am J Respir Crit Care Med 2013; 188: e13-e64.

10 Prunera-Pardell MJ, Padin-Lopez S, Domenech-Del RA, et al. Effectiveness of a respiratory rehabilitation programme in patients with chronic obstructive pulmonary disease. Enferm Clin 2018; 28: 5-12.

11 Schneeberger T, Jarosch I, Moll J, et al. Increased asthma control after a 3-week inpatient pulmonary rehabilitation program. Respir Med 2020; 165: 105930.

12 Wytrychowski K, Hans-Wytrychowska A, Piesiak P, et al. Pulmonary rehabilitation in interstitial lung diseases: a review of the literature. Adv Clin Exp Med 2020; 29: 257-264.

13 Pan American Health Organization. Rehabilitation Considerations During the COVID-19 Outbreak. https://iris. paho.org/handle/10665.2/52035 Date last updated: 19 May 2020; date last accessed: 22 March 2021.

14 [Recommendations for respiratory rehabilitation of coronavirus disease 2019 in adult]. Zhonghua Jie He He Hu Xi Za Zhi 2020; 43: 308-314.

15 Spruit MA, Holland AE, Singh SJ, et al. COVID-19: interim guidance on rehabilitation in the hospital and post-hospital phase from a European Respiratory Society and American Thoracic Society-coordinated international task force. Eur Respir J 2020; 56: 2002197.

16 Gloeckl R, Leitl D, Jarosch I, et al. Benefits of pulmonary rehabilitation in COVID-19: a prospective observational cohort study. ERJ Open Res 2021; 7: 00108-2021. 
17 Hameed F, Palatulan E, Jaywant A, et al. Outcomes of a COVID-19 recovery program for patients hospitalized withSARS-CoV-2 infection in New York City: a prospective cohort study. PM R 2021; in press.

$18 \mathrm{Al} \mathrm{CY}$, Veale D, Schoeffler M, et al. Effectiveness of pulmonary rehabilitation in COVID-19 respiratory failure patients post-ICU. Respir Physiol Neurobiol 2021; 287: 103639.

19 Liu K, Zhang W, Yang Y, et al. Respiratory rehabilitation in elderly patients with COVID-19: a randomized controlled study. Complement Ther Clin Pract 2020; 39: 101166.

20 Hermann M, Pekacka-Egli A, Witassek F, et al. Feasibility and efficacy of cardiopulmonary rehabilitation after COVID-19. Am J Phys Med Rehab 2020; 99: 865-869.

21 Daher A, Balfanz P, Cornelissen C, et al. Follow up of patients with severe coronavirus disease 2019 (COVID-19): pulmonary and extrapulmonary disease sequelae. Respir Med 2020; 174: 106197.

22 De Franca E, Junior UE, Schwingel PA, et al. Distinct phenotypes in COVID-19 may require distinct pulmonary rehabilitation strategies. Monaldi Arch Chest Dis 2020; 90. 